\title{
Comparison of Effects of Non-surgical Continuous and Intermittent Traction on Pain, Balance and Physical Function in the Treatment of Knee Osteoarthritis
}

\author{
Dong-Kyu Lee', Jin-Gyu Jeong² \\ 'Department of Physical Therapy, Sunhan Hospital, Gwangju, Republic of Korea; ${ }^{2}$ Department of Physical Therapy, Chunnam Techno University, \\ Gokseong, Republic of Korea
}

Purpose: This study examined the comparison of effects of non-surgical continuous and intermittent traction on pain, balance and physical function in the treatment of knee osteoarthritis.

Methods: A total of 30 knee osteoarthritis patients were recruited and randomized to a continuous traction group $(n=10)$, an intermittent traction group $(n=10)$, and a control group $(n=10)$. The continuous traction group and intermittent traction group received a nonsurgical continuous and intermittent knee joint traction workout five times a week, for 4 weeks. All subjects were assessed with the numeric rating scale (NRS), timed up and go test (TUGT), and Western Ontario and McMaster Universities Osteoarthritis Index (WOMAC) before and after the 4-week treatment.

Results: As a result of comparison within groups, the continuous traction group, intermittent traction group, and control group showed a significant difference for NRS, TUGT, and WOMAC after the experiment $(p<0.05)$. According to the comparison of the three groups, the continuous traction group showed a more effectively significant difference than the intermittent traction group and the control group in the balance and physical functions before and after the experiment $(\mathrm{p}<0.05)$.

Conclusion: This study showed that non-surgical continuous traction treatment was effective in improving pain, balance ability, and physical function in knee osteoarthritis patients.

Keywords: Continuous traction, Intermittent traction, Osteoarthritis

\section{INTRODUCTION}

In an aging society, osteoarthritis is a frequent musculoskeletal disorder that often occurs in the knee, hip, spine, and hand joint causing joint pain. ${ }^{1}$ Because the prevalence rate is especially high in joints that bear more weight, osteoarthritis is most common in the knee joint. ${ }^{2}$ As one of the most common aging musculoskeletal diseases, osteoarthritis degenerates the articular cartilage, forms osteophyte, and asymmetrically narrows joint space. ${ }^{3}$ Knee osteoarthritis also causes pain and the infection of the articular capsule, which limits the joint range of motion, damages muscle stability, and impairs functions in daily life. ${ }^{4}$ Imbalances that appear around the knee muscles weaken the knee muscles, decrease balance, and the joint range of motion, cause movement disorders, and increase pain. ${ }^{3,4}$
Osteoarthritis is one of the primary diseases related to the aging of the knee joint. ${ }^{5}$ It causes movement limitations and is discussed as a condition that decreases the general quality of life and contributes to anxiety, depression, decrease in self-efficacy, and chronic fatigue. ${ }^{5}$

In general, the goals of the clinical management of knee joint osteoarthritis are to provide pain relief and to maintain or improve functionality. ${ }^{6,7}$ A diverse range of surgical and non-surgical options are available for treating osteoarthritis, yet each therapeutic modality has its particular limitations and side effects. ${ }^{8}$ Furthermore, no treatments have been developed to cure knee joint osteoarthritis completely. Therefore, the goal of clinical treatments is to relieve pain, maintain or improve joint function, and reduce joint stiffening or deformation. ${ }^{89}$ Because there are difficulties in the current treatment of knee joint osteoarthritis patients to recover
Received Nov 11, 2020 Revised Dec 6, 2020

Accepted Dec 15, 2020

Corresponding author Jin-Gyu Jeong

E-mail ptj8763@cntu.ac.kr
Copylight (C2020 The Korean Society of Physical Therapy

This is an Open Access article distribute under the terms of the Creative Commons Attribution Non-commercial License (https:// creativecommons.org/license/by-nc/4.o.) which permits unrestricted non-commercial use, distribution, and reproduction in any medium, provided the original work is properly cited. 
their structures, most treatments focus on decreasing pain or improving functions. ${ }^{8,9}$ It is thus necessary to develop a treatment that improves the knee structure of knee joint osteoarthritis patients.

A method to treat a herniated intervertebral disc in the spine, traction therapy can overcome the herniation of the nucleus and reduce the pressure on a nerve root by decreasing the load on the lesion, reducing the intervertebral disc pressure, and increasing the intervertebral cavity. ${ }^{10}$ Traction therapy is currently being used to treat spine dysfunction and has been shown to relieve joint compression, improving pain and relaxing the muscle. ${ }^{10,11}$ In recent studies, traction therapy has been applied to the treatment of not only the spine but also the limb joints. ${ }^{11,12}$ The application of traction therapy using an external fixing apparatus to patients with degenerative ankle arthritis was effective for reducing pain, improving ankle joint functions, and maintaining joint space. ${ }^{12}$ Also, joint space was maintained even after the external fixing apparatus was removed. ${ }^{12,13}$ In a recent study, a traction therapy on patients with knee osteoarthritis using a surgical method also showed positive results. ${ }^{14}$ Traction therapy using the surgical method can expand the joint space of the limbs. ${ }^{15}$ Also, it has been reported that traction therapy on knee joint effectively increases joint space, maintains expansion, increases cartilage thickness, reduces the lost skeletal region, and improves joint function. ${ }^{14,15}$ However, traction therapy using surgical methods takes a long time for patients to recover and can increase their psychological anxiety if they have difficulty in daily. ${ }^{12-14}$ Traction therapy methods that are applied not only to the spine but also to the limb joints include continuous traction therapy and intermittent traction therapy, and several studies have been reported on their positive and negative effects. ${ }^{16,17}$ However, there is an ongoing controversy over the effectiveness of continuous traction therapy and intermittent traction therapy. ${ }^{11}$ Furthermore, few studies are comparing non-surgical continuous traction therapy and intermittent traction therapy. Therefore, this study aims to identify the effects of non-surgical continuous and intermittent traction on pain, balance and physical function in the treatment of knee osteoarthritis

\section{METHODS}

\section{Subjects}

A total of 30 older people aged $\geq 65$ years, who sufficiently understood the purpose and contents of this study and agreed voluntarily, participated in this study. They are knee osteoarthritis patients who had a full range of motion in the knee joints and had not received surgical treatment on their legs. They were randomly divided into three groups: continuous traction group, intermittent traction group, and control group. The subjects will be included if they are over 65 years, have a Kellgren-Lawrence (K-L) grade of $>2$, not currently exercising, and able to understand the exercise. The subjects will be excluded if they had received surgery on the knee joint and had restrictions in maintaining balance due to central nervous system disease or visual sense disease. Before the experiment, all participants were explained about the contents and purpose of the study and submitted written informed consent. The institutional human ethics committees approved the study. The study also included a signed consent form, according to the ethical standards of the declaration of Helsinki. Table 1 shows the general characteristics of the subjects.

\section{Interventions}

Both the continuous traction group and the intermittent traction group received a continuous and intermittent knee joint traction workout five times a week, for 4 weeks. In this study, we performed a traction therapy by referring to, modifying, and supplementing the study by Alpayci et al. ${ }^{18}$ according to the treatment environment. Continuous traction was conducted on the knee joint continuously for 20 minutes, whereas intermittent traction therapy was performed four times repeatedly on the knee joint for 5 minutes each, followed by 5 -minutes rest. The participants were asked to bend their hip and knee joints at $60^{\circ}$ in the supine position. The tibia and thigh were secured with a strap, and intermittent or continuous knee joint traction treatment was applied to tow the tibia in the cephalocaudal direction. The force that was applied by the traction was approxi-

Table 1. General characteristics of the subjects

\begin{tabular}{|c|c|c|c|c|}
\hline & CTG $(n=10)$ & ITG $(n=10)$ & $C G(n=10)$ & $F(p)$ \\
\hline $\operatorname{Sex}(M / F)$ & $5 / 5$ & $4 / 6$ & $6 / 4$ & \\
\hline Age (yr) & $65.8 \pm 5.1$ & $67.7 \pm 4.4$ & $66.3 \pm 4.5$ & $0.444(0.646)$ \\
\hline Height (cm) & $164.6 \pm 6.4$ & $164.8 \pm 3.6$ & $163.3 \pm 7.4$ & $0.183(0.833)$ \\
\hline Weight (kg) & $60.6 \pm 6.1$ & $62.3 \pm 4.0$ & $61.1 \pm 6.5$ & $0.238(0.790)$ \\
\hline K-L grade (\%) & $2.6 \pm 0.7$ & $2.7 \pm 0.5$ & $2.5 \pm 0.5$ & $0.300(0.743)$ \\
\hline
\end{tabular}

Values are presented as mean \pm standard deviation.

K-L grade: Kellgren-Lawrence grade, CTG: Continuous traction group, ITG: Intermittent traction group, CG: Control group. 


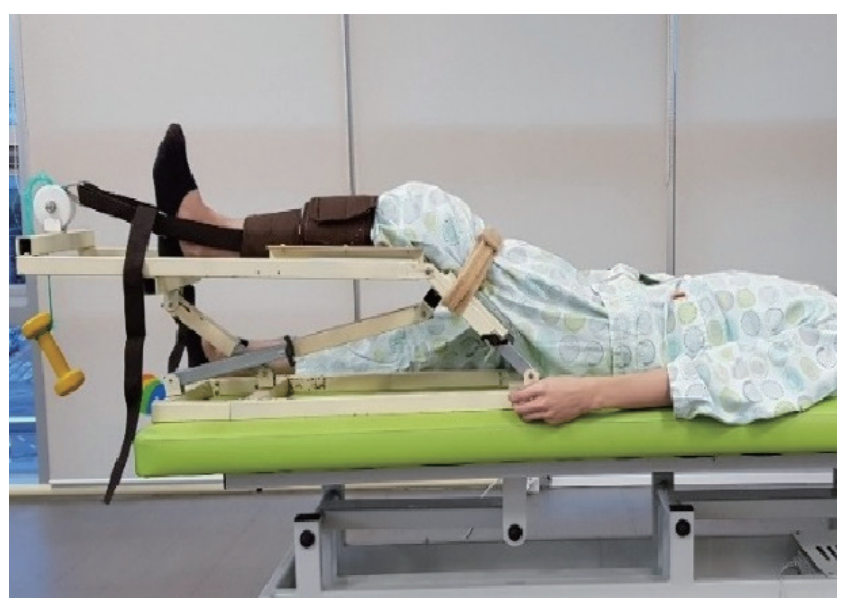

Figure 1. Non-surgical Continuous and Intermittent Traction.

mately equal to $6 \%$ of the participant's weight (Figure 1). The control group received general physical therapy five times for 4 weeks: superficial heat therapy (20 minutes), deep heat therapy (5 minutes), and electric therapy (20 minutes).

\section{Experimental methods}

From all the patients, the pain was measured using the numeric rating scale (NRS), which evaluates the intensity of subjective pain: NRS ranging from 0 (no pain) to 10 (most severe pain).

Balance and walking ability were measured using the timed up and go test (TUGT). In the TUGT, we measured the time when the patient 1) stands up from the armchair, 2) walks $3 \mathrm{~m}$, and 3) returns to the armchair to sit down.

Physical function was measured using the Western Ontario and McMaster Universities Osteoarthritis Index (WOMAC) scale, which measures specific diseases, personal health management, and physical conditions. It is a clinically important functional evaluation tool for assessing pain, stiffening, and physical function in patients with osteoarthritis and coxarthritis. In the WOMAC, the physical function can be measured by asking the patient directly to complete a self-reported questionnaire. The index consists of 24 categories: 5 categories for pain, 2 categories for stiffening, and 17 categories for physical function. All questions are scored on a scale of $0-4(0=$ none, $1=$ mild, $2=$ moderate, $3=$ severe, $4=$ very severe $)$, with 4 representing the worst pain, stiffness, and functional limitations. We recorded the NRS, TUGT score, and WOMAC score before and after the 4-week treatment.

\section{Statistical analysis}

We used SPSS version 19.0 (IBM, Chicago, IL, USA) for data analysis and descriptive statistics for the analysis of the patients' general characteristics. By using the Shapiro-Wilk test, we performed the normality tests. The pre-experiment homogeneity test was conducted for patients' general characteristics; the dependent variables were analyzed by one-way ANOVA. We used the paired t-test to respectively verify the difference between pre-test and post-test mean values within the group. To compare the differences in variation between the groups, we performed one-way ANOVA. To explain the differences between the groups after the test, we used the least-squares difference method for post-hoc analysis. The statistical significance level was set at $\alpha=0.05$.

\section{RESULTS}

\section{Pain}

The results of repeated the continuous traction group exhibited a significant difference as its NRS score, which decreased from $6.06 \pm 0.88$ before treatment to $5.06 \pm 0.79$ after treatment $(\mathrm{p}<0.05)$ (Table 2). The intermittent traction group exhibited a significant difference as its NRS score, which decreased from $6.06 \pm 0.88$ before treatment to $5.06 \pm 0.79$ after treatment $(\mathrm{p}<0.05)($ Table 2$)$. The control group exhibited a significant difference as its NRS score, which decreased from $6.06 \pm 0.88$ before treatment to $5.06 \pm 0.79$ after treatment $(\mathrm{p}<0.05)$ (Table 2). For difference in pain before and after the experiment among the three groups, the continuous traction group had a more effective significant result than the intermittent traction group and the control group $(\mathrm{p}<0.05)$ (Table 2).

\section{Balance and walking ability}

The continuous traction group exhibited a significant difference as its TUGT score, which decreased from $6.06 \pm 0.88$ before treatment to $5.06 \pm$ 0.79 after treatment $(\mathrm{p}<0.05)$ (Table 2). The intermittent traction group exhibited a significant difference as its TUGT score, which decreased from $6.06 \pm 0.88$ before treatment to $5.06 \pm 0.79$ after treatment $(\mathrm{p}<0.05)$ (Table 2). The control group exhibited a significant difference as its TUGT score, which decreased from $6.06 \pm 0.88$ before treatment to $5.06 \pm 0.79$ after treatment $(\mathrm{p}<0.05)$ (Table 2$)$. For balance and walking ability before and after the experiment among the three groups, the continuous traction group had a more effective significant difference than the intermittent traction group and the control group $(\mathrm{p}<0.05)$ (Table 2$)$. 
Table 2. Changes in NRS, TUGT, WOMAC in this study

\begin{tabular}{|c|c|c|c|c|c|c|}
\hline & CTG $(n=10)$ & ITG $(n=10)$ & $C G(n=10)$ & $\mathrm{F}$ & $\mathrm{p}$ & Post-hoc \\
\hline \multicolumn{7}{|l|}{ NRS } \\
\hline Pre & $7.40 \pm 1.26$ & $7.90 \pm 0.87$ & $8.30 \pm 0.67$ & & & \\
\hline Post & $2.70 \pm 1.05$ & $5.00 \pm 1.05$ & $6.00 \pm 0.94$ & & & \\
\hline Difference & $-4.70 \pm 1.56^{*}$ & $-2.90 \pm 0.99^{\star}$ & $-2.30 \pm 1.05^{\star}$ & 10.248 & $<0.001$ & $\mathrm{CTG}>\mathrm{ITG}, \mathrm{CTG}>\mathrm{CG}$ \\
\hline $\mathrm{t}$ & 9.845 & 9.222 & 6.866 & & & \\
\hline $\mathrm{p}$ & $<0.001$ & $<0.001$ & $<0.001$ & & & \\
\hline \multicolumn{7}{|l|}{ TUGT } \\
\hline Pre & $11.10 \pm 1.10$ & $10.60 \pm 1.64$ & $11.30 \pm 1.25$ & & & \\
\hline Post & $6.90 \pm 1.56$ & $7.90 \pm 0.99$ & $8.80 \pm 1.22$ & & & \\
\hline Difference & $-4.20 \pm 1.31^{*}$ & $-2.70 \pm 1.82^{*}$ & $-2.50 \pm 1.43^{*}$ & 3.631 & 0.040 & $C T G>\mid T G, C T G>C G$ \\
\hline t & 10.088 & 4.669 & 5.514 & & & \\
\hline $\mathrm{p}$ & $<0.001$ & $<0.001$ & $<0.001$ & & & \\
\hline \multicolumn{7}{|l|}{ WOMAC } \\
\hline Pre & $43.70 \pm 2.40$ & $42.80 \pm 3.25$ & $44.50 \pm 1.71$ & & & \\
\hline Post & $28.80 \pm 3.32$ & $32.80 \pm 3.19$ & $38.40 \pm 6.00$ & & & \\
\hline Difference & $10.688^{*}$ & $7.032^{*}$ & $3.429^{*}$ & 8.180 & 0.002 & $\mathrm{CTG}>\mid \mathrm{TG}, \mathrm{CTG}>\mathrm{CG}$ \\
\hline $\mathrm{t}$ & $-14.90 \pm 4.40$ & $-10.00 \pm 4.49$ & $-6.10 \pm 5.62$ & & & \\
\hline$p$ & $<0.001$ & $<0.001$ & 0.008 & & & \\
\hline
\end{tabular}

Values are presented as mean \pm standard deviation.

NRS: Numeric Rating Scale, TUGT: Timed Up and Go Test, WOMAC: Western Ontario and McMaster universities osteoarthritis, CTG: Continuous traction group, ITG: Intermittent traction group, CG: Control group. ${ }^{*} p<0.05$.

\section{Physical function}

The continuous traction group exhibited a significant difference as its WOMAC score, which decreased from $47.20 \pm 1.65$ before treatment to $25.33 \pm 2.38$ after treatment $(\mathrm{p}<0.05)$ (Table 2$)$. The intermittent traction group exhibited a significant difference as its WOMAC score, which decreased from $47.20 \pm 1.65$ before treatment to $25.33 \pm 2.38$ after treatment $(\mathrm{p}<0.05)$ (Table 2). The control group exhibited a significant difference as its WOMAC score, which decreased from $44.50 \pm 1.71$ before treatment to $38.40 \pm 6.00$ after treatment $(\mathrm{p}<0.05)$ (Table 2). For physical functions before and after the experiment among the three groups, the continuous traction group showed a more effective, significant difference than the intermittent traction group and the control group $(\mathrm{p}<0.05)$ (Table 2$)$.

\section{DISCUSSION}

Traction is a physical therapy modality used commonly for spinal pain..$^{10,11}$ However, experience in traction treatment for knee osteoarthritis is very limited, and the long-term effects of such a method are unknown. Although previous studies performed traction therapy on ankle and knee osteoarthritis, most of them used the surgical method. ${ }^{12-14}$ Thus, this study aimed to examine the effects of applying non-surgical traction therapy for
4 weeks to knee osteoarthritis patients according to pain, balance and walking ability, and physical function. We also aimed to determine which traction method is more effective for knee osteoarthritis patients by dividing the traction therapy into continuous traction and intermittent traction. Pain is the main symptom in knee osteoarthritis. ${ }^{4}$ Intema et al. ${ }^{14}$ showed that traction therapy using an external surgical fixing device reduced the pain of knee osteoarthritis patients. In our study, we observed significant improvements in pain for continuous traction group and intermittent traction group at week 4 , compared with baseline. Because this study performed traction using a non-surgical method, returning to daily life will be much faster. The results of this study were consistent with the study by Choi and Lee, ${ }^{19}$ which they applied non-surgical traction to patients with degenerative knee osteoarthritis in reducing the pain. Surgical traction uses drugs to relieve the pain, for example, in Sudeck's atrophy; however, the non-surgical method will have positive effects on osteoarthritis patients because traction can be applied without the use of drugs. ${ }^{12}$ This study confirmed that continuous traction treatment was more effective for reducing and improving pain than intermittent traction treatment. The effectiveness of continuous traction treatment on the pain may be performed by flexing of joint structures, reducing hypoxia and subchondral pressure by increasing circulation, and inhibiting pro-inflammatory 
cytokines by decreasing intra-articular stress. ${ }^{18}$ Because joint cartilage is aneural, the pain in knee osteoarthritis patients results from periarticular tissues and intra-articular tissues outside the cartilage. ${ }^{18}$ The increase in joint space width might be associated with the formation of fibrous tissue with a consequent change of load transfer in the joint and a decrease in pain. ${ }^{12}$ The effects of continuous traction may provide a strong stimulus to connective tissue, resulting in pain relief.

Improving a patient's ability to walk is important as it helps in maintaining independence to perform activities of daily living. ${ }^{20}$ In knee osteoarthritis patients, the balance and walking ability is impaired because of the pain and stiffness in the joint. ${ }^{4}$ Hence, the walking ability of the patients was improved when non-surgical traction was applied to them. ${ }^{21}$ Because the TUGT value decreased significantly before and after the experiment when mechanical traction therapy was applied to the patients, the study of Lee et al. ${ }^{22}$ could be supported by our results. Khademi-Kalantari et al. ${ }^{21}$ and Lee et al. ${ }^{22}$ applied non-surgical continuous traction treatment to knee osteoarthritis patients and showed positive effects on improving their balance and walking ability, which was consistent with the results of this study. Joint traction is also a good stimulus for mechanoreceptors located in the knee structures, such as joint capsule and ligaments. 18 The widening of the joint space via traction may reduce intra-articular stress. ${ }^{18,21}$ Previous studies reported cartilage regeneration by joint traction. ${ }^{18,23}$ Our traction methods may provide instrument-assisted joint and soft tissue mobilization and manipulation and may decrease the knee osteoarthritis symptoms. After continuous traction in knee osteoarthritis patients, the decrease in pain improves their physical functions and enhances their balance and walking ability.

In this study, applying non-surgical traction for 4 weeks to knee osteoarthritis patients improved their physical functions. Intema et al. ${ }^{14}$ reported that applying traction using a surgical method for 8 weeks had a positive effect on improving the physical functions of knee osteoarthritis patients. Choi and Lee ${ }^{19}$ reported that physical functions were improved as a result of applying non-surgical traction for 4 weeks to patients with degenerative knee arthritis. This showed that non-surgical traction recovered patients' physical functions in a shorter period than surgical traction, and thus, this supported the results of this study. A mechanism of this superior effect in the traction groups may be the mobilization of joint structures (e.g., joint capsule, ligament, muscle, and tendon). ${ }^{18,23}$ By traction, the stimulation of the mechanoreceptors in these structures may play a critical role in the efficacy of joint stiffness and physical function. ${ }^{18,23}$ This study confirmed that continuous traction showed more significant improvement in the physical functions of patients than intermittent traction. However, Khademi-Kalantari et al. ${ }^{21}$ reported that continuous traction rather than intermittent traction improved physical functions more significantly. Also, Choi and Lee ${ }^{19}$ reported that continuous traction had a positive effect on improving the physical functions of patients with degenerative knee osteoarthritis. On the basis of these studies, continuous traction is considered more helpful than intermittent traction in improving the physical functions of knee osteoarthritis patients.

On the basis of these results, continuous traction therapy is thought to improve pain, balance and walking ability and physical function in knee osteoarthritis patients; in this study, all of which were confirmed to improve after therapy in knee osteoarthritis patients.

There are a few limitations to this study. It is difficult to generalize our results because of the small number of participants. The most effective methods of continuous traction application for knee osteoarthritis are unclear. Perhaps, the application of traction with longer periods or using greater weights is required to obtain more positive effects on the symptoms of knee osteoarthritis; however, the possible side effects should be considered. Furthermore, we could not conduct a follow-up study to investigate the long-term effects of continuous traction after treatment termination. To generalize the results of our study, we will conduct a longterm follow-up study using a larger number of knee joint arthritis patients in the future. Hence, this research has huge significance as it has applied the non-surgical continuous traction on pain, balance and physical function in the treatment of knee osteoarthritis.

\section{REFERENCES}

1. Keenan AM, Tennant A, Fear J et al. Impact of multiple joint problems on daily living tasks in people in the community over age fifty-five. Arthritis Rheum. 2006;55(5):757-64.

2. Juhl C, Christensen R, Roos EM et al. Impact of exercise type and dose on pain and disability in knee osteoarthritis: A systematic review and meta-regression analysis of randomized controlled trials. Arthritis Rheumatol. 2014;66(3):622-36.

3. Lories RJ, Luyten FP. The bone-cartilage unit in osteoarthritis. Nat Rev Rheumatol. 2011;7(1):43-9.

4. Baliunas AJ, Hurwitz DE, Ryals AB et al. Increased knee joint loads during walking are present in subjects with knee osteoarthritis. Osteoarthr Carti. 2002;10(7):573-9.

5. Turner JA, Ersek M, Kemp C. Self-efficacy for managing pain is associated with disability, depression, and pain coping among retirement community residents with chronic pain. J Pain. 2005;6(7):471-9.

6. Migliore A, Procopio S. Effectiveness and utility of hyaluronic acid in osteoarthritis. Clin Cases Miner Bone Metab. 2015;12(1):31-3. 
7. McKay C, Prapavessis H, Doherty T. The effect of a prehabilitation exercise program on quadriceps strength for patients undergoing total knee arthroplasty: a randomized controlled pilot study. PMR. 2012;4(9):64756.

8. Hochberg MC, Altman RD, April KT et al. American college of rheumatology 2012 recommendations for the use of nonpharmacologic and pharmacologic therapies in osteoarthritis of the hand, hip, and knee. Arthritis Care Res. 2012;64(4):465-74.

9. Zhang W, Moskowitz RW, Nuki G et al. OARSI recommendations for the management of hip and knee osteoarthritis, Part II: OARSI evidence-based, expert consensus guidelines. Osteoarthr Cartil. 2008; 16(2):137-62.

10. Park SH, Kim CS, Lee DG et al. The short term effects of the decompression (KNX 7000(R)) and traction device on pain in patients with chronic low back pain with or without radicular pain. J Kor Phys Ther. 2011;23 (5):29-34.

11. Kwon WA, Ma SY, Hwang YT. The effects of intermittent traction and manual traction on lumbar herniated disc. J Kor Phys Ther. 2009;21 (3):9-16.

12. Ploegmakers JJ, Van Roermund PM, Van Melkebeek J et al. Prolonged clinical benefit from joint distraction in the treatment of ankle osteoarthritis. Osteoarthr Cartil. 2005;13(7):582-8.

13. Marijnissen ACA, Van Roermund PM, Van Melkebeek J et al. Clinical benefit of joint distraction in the treatment of severe osteoarthritis of the ankle: Proof of concept in an open prospective study and in a randomized controlled study. Arthritis Rheum. 2002;46(11):2893-902.

14. Intema F, Van Roermund PM, Marijnissen AC et al. Tissue structure modification in knee osteoarthritis by use of joint distraction: An open 1-year pilot study. Ann Rheum Dis. 2011;70(8):1441-6.

15. Van Valburg AA, Van Roermund PM, Marijnissen AC et al. Joint distraction in treatment of osteoarthritis: A two-year follow-up of the ankle. Osteoarthr Cartil. 1999;7(5):474-9.

16. Sari H, Akarirmak U, Karacan I et al. Computed tomographic evaluation of lumbar spinal structures during traction. Physiother Theory Pract. 2005;21(1):3-11.

17. Rattanatharn R, Sanjaroensuttikul N, Anadirekkul P et al. Effectiveness of lumbar traction with routine conservative treatment in acute herniated disc syndrome. J Med Assoc Thai. 2004;87(2):272-7.

18. Alpayci M, Ozkan Y, Yazmalar L et al. A randomized controlled trial on the efficacy of intermittent and continuous traction for patients with knee osteoarthritis. Clin Rehabil. 2013;27(4):347-54.

19. Choi MS, Lee DK. The effect of knee joint traction therapy on pain, physical function, and depression in patients with degenerative arthritis. J Kor Phys Ther. 2019;31(5):317-21.

20. Stratford PW, Kennedy DM, Woodhouse LJ. Performance measures provide assessments of pain and function in people with advanced osteoarthritis of the hip or knee. Phys Ther. 2006;86(11):1489-96.

21. Khademi-Kalantari K, Mahmoodi Aghdam S, Akbarzadeh Baghban A et al. Effects of non-surgical joint distraction in the treatment of severe knee osteoarthritis. J Bodyw Mov Ther. 2014;18(4):533-9.

22. Lee NY, Kwon CS, Kim SY. The effect of mechanical traction on pain and physical function in patients with knee osteoarthritis. Phys Ther Korea. 2015;22(3):23-32.

23. Goh EL, Lou WCN, Chidambaram S et al. Joint distraction for knee osteoarthritis: protocol for a systematic review and meta-analysis. Syst Rev. 2018;7(1):162-5. 